\title{
Telemedicine Applications for the Evaluation of Patients with Non-Acute Headache: A Narrative Review
}

\author{
Constantinos Dean Noutsios' \\ Virginie Boisvert-Plante (iD) \\ Jordi Perez ${ }^{2,3}$ \\ Jonathan Hudon ${ }^{2-6}$ \\ Pablo Ingelmo iD ${ }^{3,4}$
}

'Faculty of Medicine, McGill University, Montreal, QC, Canada; ${ }^{2}$ Alan Edwards Pain Management Unit. Montreal General Hospital, McGill University Health Center, Montreal, QC, Canada; ${ }^{3}$ Alan Edwards Centre for Pain Research, McGill University, Montreal, QC, Canada; ${ }^{4}$ Edwards Family Interdisciplinary Complex Pain Centre, Montreal Children's Hospital, McGill University Health Center, Montreal, QC, Canada; ${ }^{5}$ Faculty Lecturer (Clinical), Department of Family Medicine, Faculty of Medicine, McGill University, Montreal, QC, Canada; ${ }^{6}$ Division of Secondary Care,

Department of Family Medicine, McGill University Health Centre, Montreal, QC, Canada
Correspondence: Pablo Ingelmo Edwards Family Interdisciplinary Complex Pain Centre. Montreal Children's Hospital, McGill University Health Centre, A.02.3525-I00I Boul. Decarie, Montreal, QC, H4A 3JI, Canada

Tel + I 5I4 4I24448

Fax + I 514412 434I

Email pablo.ingelmo@mcgill.ca

\begin{abstract}
The COVID-19 pandemic has spurred a hasty transition to virtual care but also an abundance of new literature highlighting telehealth's capabilities and limitations for various healthcare applications. In this review, we aim to narrate the current state of the literature on telehealth applied to migraine care. First, telemedicine in the context of non-acute headache management has been shown to produce non-inferior patient outcomes when compared to traditional face-to-face appointments. The assignment of patients to telehealth appointments should be made after referring more urgent cases to dedicated in-person clinics. During the virtual appointment, physicians can ask their patients about the "3 F's" in order to perform a thorough assessment of their headaches: frequency of headache days, frequency of acute medication usage and functional impairment. Clinical assessment scores that have been studied and deemed feasible for telemedicine, safe and efficient include the HIT-6, VAS and MIDAS scores. Although MIDAS was found to be redundant and inadequate to use on a daily basis, we suggest that it can be useful in periodic remote follow-up appointments. Additionally, several mobile health apps have been studied including Migraine Buddy, Migraine Coach and Migraine Monitor. All of these are appropriate for use in telemedicine when combined with an adequate trial period with Migraine Buddy being rated the highest, as it captures the most detailed clinical picture. High satisfaction rates have been reported for virtual headache management which were shown to be equal to in-person consults. These are based on patients' perceived increase in convenience due to avoided travel time, less disruption of their daily routine and feeling more comfortable in the environment of their choice. Despite this, limitations such as technological knowledge, access to videoconferencing modalities and having a more impersonal consultation with the physician may hinder some patients from adopting this service.
\end{abstract}

Keywords: telehealth, telemedicine, headache, evaluation, mHealth, tele-assessment

\section{Introduction}

The International Headache Society (IHS) defines headache as a heterogeneous group of neurological disorders including migraine, tension-type headache and trigeminal autonomic cephalalgias which are all primary disorders. Headaches can also be secondary to other pathologies or arise from of cranial nerve lesions. ${ }^{1}$ The most prevalent headache is migraine, which was ranked as the second highest cause of age-standardized disability worldwide in the 2016 Global Burden of Disease Study. ${ }^{2}$ Given the latter, effort towards improving care and access to care is imperative. 
We are living in a time of emerging digital technology which has allowed health services to evolve. Telemedicine is defined broadly as the real-time delivery of health services at a distance via means of digital technology. ${ }^{3}$ Telehealth and electronic health (eHealth) are related definitions, the latter encompassing mobile health (mHealth) which includes the use of smartphones, applications and wearables for medical purposes. ${ }^{3}$ Within the current COVID-19 pandemic climate, there has been a hasty shift of clinical consultations towards telemedicine visits. ${ }^{4}$ Face-to-face consultations for patients with nonacute headaches are being replaced by videoconference meetings or telephone encounters, bringing along their own set of advantages and limitations. ${ }^{5}$ Almost all medical specialties have been impacted by the rapid improvements in telemedicine services and their potential applications. ${ }^{6}$ Telemedicine for the headache patient is an excellent alternative since there is less emphasis for repeated routine physical exams. Clinical tasks such as reviewing history, medications, treatment response and education are easily performed via telemedicine approaches. ${ }^{7,8}$

With respect to headache management and treatment, there have been several studies reporting on the implementation of videoconference consultations as well as mHealth technologies aimed at improving communication between patient and physician. ${ }^{9,10}$ However, due to rapid developments in the field of telemedicine, reviews describing structured telemedicine consultations and highlighting useful mobile tools for headache patients are lacking. Therefore, the goal of this narrative review is to summarize the available research in delivery of headache management by means of telemedicine. We will focus on how to select patients suitable for a telemedicine interview, what components of the history must be included in a consultation as well as what assessment measures are proven to be useful in the literature. Additionally, we will describe mHealth tools available to physicians and compare traditional appointments to virtual consultations.

\section{Methods}

The PubMed database was searched for available journal articles from January 2015 to December 2020 published in the English language. Our search was limited to this period to include primarily new telemedicine technology. A research question in the population, intervention, comparison and outcome (PICO) format was generated to guide the literature search and develop a screening process for relevant articles. The question was: In patients with non-acute headache, are consultations delivered via telemedicine feasible and comparable to in-person appointments in terms of clinical outcomes and patient satisfaction? The following keywords were used: telehealth, telemedicine, tele-assessment, teleconsultation, mHealth, eHealth, migraine and headache. These keywords were combined to use the following nested search: ("telemedicine"[MeSH] OR "telehealth" OR "teleassessment" OR "teleconsultation" OR "eHealth" OR "mHealth") AND ("headache" OR "migraine"). A total of 69 citations were found for which titles and abstracts were screened for relevance and consideration for thorough review. We excluded 57 articles due to lack of relevance to our topic. The remaining 12 full-text articles were examined as well as their reference list which yielded 6 additional articles. Finally, 13 additional references were individually selected during manuscript preparation to provide clarification on certain topics, yielding a total of 31 references.

\section{Telemedicine Modalities}

Telemedicine appointments are usually performed over a personal videoconferencing system or a software application installed on a desktop or smartphone. It is best performed in a calm environment where the patient and the clinician feel comfortable discussing personal information. The room should have proper lighting and, ideally, both parties should have a high-speed internet service. If the patient does not have access to a device or should technical difficulties arise during the videoconference, a telephone consultation could be an alternative for a new consultation or a follow-up, bearing in mind that body language cannot be read, nor physical exam performed. There are currently no studies comparing telephone to videoconference calls in the context of headache consultations and management specifically. However, a systematic review comparing these two modalities in other healthcare contexts including pediatrics, stroke, neurosurgery, depression and smoking cessation revealed that videoconferencing had superior provider-related outcomes, greater diagnostic accuracy, improved decision-making accuracy and result in fewer medications errors. ${ }^{11}$ Given this, we suggest that new patient consultations should be scheduled for an audio-visual call rather than a telephone call.

\section{Patient Selection and Initial Classification}

A successful triaging process at a dedicated pediatric neurologic clinic has been demonstrated effective in transitioning to telemedicine approaches. ${ }^{12}$ Their success lied mainly 
in their initial classification step, where new-patient referrals were first screened by a physician who would automatically direct the most urgent cases to a dedicated clinic. Most new and established patients were then scheduled for an audio-visual appointment or a phone appointment if they did not have access to a smartphone or a computer. While only $5 \%$ of the patients initially seen over telemedicine needed an in-person appointment, the majority could be managed by telemedicine exclusively. ${ }^{12}$ Reasons for emergent in-person consult included possible seizure, abnormal movements, sensory disturbances, as well as metabolic, facial nerve and neuromuscular disorders. These results further emphasize the need for initial screening. This structure could be implemented in the context of a chronic pain or migraine clinic where a healthcare professional could screen new referrals in order to promptly identify the most urgent cases requiring immediate in-person consultation. We believe that existing patients seeking an appointment due to an acute flare-up can be managed in a similar fashion. When screening patients, it is important for physicians to be vigilant for red flags pointing to a secondary headache and refer these patients for face-to-face consultations.? These include new-onset severe headache, worsening of existing headache (frequency or severity), change in the character of an existing headache as well as presence of focal neurological abnormalities or constitutional symptoms.

The frequency and pattern of headache episodes mandate different clinical approaches with regards to follow-up visits. A classification of patients within 4 separate groups has been proposed with views of identifying the most urgent cases and guiding treatment choice. ${ }^{7}$ These groups include infrequent $(<$ 4 headaches/month), episodic (4-14 headaches/month), chronic ( $>15$ headaches/month) and newly diagnosed. Patients with infrequent headaches mostly need reassurance with minimal intervention. Episodic and chronic cases should be assessed regularly with ad-hoc visits to optimize their therapy and prevent or minimize ER visits. Finally, newly diagnosed patients require viable treatment options and adequate educational support. Physicians should also tailor their treatment plan according to the patients' migraine frequency, which may consist of acute symptomatic relief, lifestyle modifications, prophylactic treatment as well as educational support. $^{?}$

\section{Components of Patient Interview}

A modified 2-round Delphi panel investigated what questions would be the most pertinent to include in a digital migraine tracker. ${ }^{13}$ This analysis identified the " 3 F's" as the most essential components of the interview: frequency of headache days, frequency of acute medication usage and functional impairment. The panel recommended that the interview should address the headaches themselves (occurrence, symptoms, daily prevention and medication), the triggers (concentration, rest and unusual activities) and lastly, the burden of headaches as indicated by the levels of absenteeism and productivity, in that order. Lastly, a prospective, open-labelled, non-inferiority randomized control trial (RCT) used a 28-item questionnaire to assess patients' headache burden and satisfaction with the consultation in either a face-to-face or telemedicine setting. ${ }^{14}$ This questionnaire was given during the first appointment, and then 3 months later for comparison, touching on several important areas, namely:

1. Patient satisfaction with consultation

2. Subjective change in headache frequency and intensity

3. Number of headache days per month

4. Headache intensity as measured by the visual analog scale (VAS) and the Headache Impact Test (HIT-6)

5. Impact on lifestyle parameters (sick leave, employment)

6. Use of medication, including painkillers and triptans

7. Recall of headache diagnosis and compliance with said diagnosis

In summary, when performing their own telemedicine interview for headache patients, physicians may find it helpful to incorporate the 3 F's. They might also choose to use a questionnaire or incorporate parts of it in their follow-ups. Lastly, they can use satisfaction surveys to identify areas of potential improvement.

\section{Assessment Scores}

Clinical assessment scores are also useful in the context of telemedicine when assessing the functional disability of a patient suffering from non-acute headaches. Three studies identified the use of HIT- 6 and VAS ${ }^{14-16}$ scores and two studies identified the use of Migraine Disability Assessment (MIDAS) ${ }^{8,17}$ scores to be feasible and appropriate for telemedicine in the context of headache management. For example, Müller et al published two studies in which an RCT was conducted allocating two groups of patients suffering from chronic headache to a telemedicine and traditional appointment. ${ }^{14,15}$ Outcome variables used, among others, 
were the HIT-6 and VAS at the 3-month and 12-month mark. No statistical difference was found between telemedicine and traditional consultations for both scores at both time points, suggesting that using the HIT-6 and VAS in a telemedicine context for non-acute headaches was feasible, safe and efficient. Friedman et al conducted a small, randomized trial using the MIDAS score as one of the clinical outcomes to compare effectiveness between telemedicine and traditional groups. ${ }^{8}$ They found that there was no statistical difference between scores of both groups at 12 months, making MIDAS feasible and effective to use for follow-up migraine care. Similarly, Huguet et al worked on the development of an electronic migraine diary app that would incorporate questions from the MIDAS test. ${ }^{17}$ They measured patient outcomes by following the Initiative on Methods, Measurement, and Pain Assessment in Clinical Trials (IMMPACT) recommendations. They concluded that although MIDAS is useful, it is redundant and inadequate to use on a daily basis, such as in the context of a daily headache diary app. Despite this, we suggest that MIDAS can be useful in periodic follow-up appointments over the phone or videoconference. It is also important to mention that the aforementioned studies used these scores as outcome measures to compare the efficacy of telemedicine versus traditional consultations or to establish non-inferiority of telemedicine. Therefore, physicians can confidently use these scores in assessing the severity of their patients' headaches.

\section{Virtual Neurologic Exam}

When assessing patients suffering from headaches, physicians deem it relevant to perform a focused physical exam as well as a neurological exam. Although it might not be possible to conduct a full neurological examination, it is feasible to perform parts of it remotely. Herein, we summarize how it is conducted, as described by VerduzcoGutierrez et $\mathrm{al}^{18} \mathrm{Al}$ Hussona et $\mathrm{al}^{19}$ and Robblee in an American Headache Society video. ${ }^{20}$

The evaluation of the mental status includes assessment of alertness, orientation in time, space and person and ability to focus on a task or recall a recent event. The rate of speech, word choice, fluency and voice volume should also be observed. Despite not being able to conduct a true assessment of tone, ${ }^{20}$ the clinician can observe for voluntary and involuntary movements, muscle cocontraction and assess if there is posturing with position changes. Neuromuscular and motor evaluation includes inspection of the muscle bulk in the upper and lower limbs, presence of bradykinesia or abnormal movements at rest including tremor, dystonia and clonus. Physicians may also wish to instruct the patient to perform a rapid finger tapping test, pronator drift and forearm roll. As a basic assessment of strength, they can instruct their patients to perform squats and unilateral heel raises. Heel and toe walking can be performed to assess for dorsiflexion/plantar flexion strength.

Coordination can be assessed by instructing the patient to perform rapid alternating hand movements, finger-toobject testing with available targets (eg, edge of computer screen) or heel-to-shin testing. Proprioception can be evaluated by instructing the patient to perform the Romberg and tandem walking tests if the physician deems it safe. Sensation is assessed by having the patient or an assistant lightly touch the different dermatomes and report on abnormal sensation. Similarly, pinprick and temperature sensation can be assessed with the sharp edge of a pencil or with ice, respectively. When concerned about an upper motor neuron lesion, physicians can perform the Babinski sign over telemedicine, although it is noted to be unreliable. $^{20}$ For this test, physicians can instruct a family member to use the sharp end of a pen, with the patient's plantar side of their foot close to the camera. Lastly, the physician can assess patients' gait as well as tandem gait by asking them to walk back and forth in front of the camera. Table 1 summarizes how to virtually assess the cranial nerves.

Limitations in performing a virtual neurologic exam include the inability to perform a fundoscopic exam, a true assessment of tone or assess deep tendon reflexes and muscle power. ${ }^{20}$ Despite this, the virtual neurologic exam is sufficient for the context of headache assessment.

\section{mHealth Tools}

Mobile health (mHealth) allows for point-of-care data collection and in the headache context, can include headache diaries and migraine trigger trackers. These tools can be used to increase adherence to treatment by allowing medication monitoring and by providing support and motivation. Smartphones are now widely accessible which makes use of mHealth tools feasible for most patients. When choosing a medical application, those having a website platform as well as a separate interface for physician and patient are preferred. ${ }^{21}$ They have been found to facilitate communication between patients and physicians and to increase patient accountability. ${ }^{15}$ Electronic diaries are the most prominent mHealth tools 
Table I Performing an Examination of the Cranial Nerves via Telemedicine ${ }^{\mathrm{a}}$

\begin{tabular}{|c|c|}
\hline $\begin{array}{l}\text { Cranial } \\
\text { Nerve }\end{array}$ & Virtual Assessment \\
\hline CN I & $\begin{array}{l}\text { - Given that } \mathrm{CN} \text { I is seldom examined, abnormalities in smell can be screened for in the patient's history. } \\
\text { - If the physician wishes to examine it, they can have patients identify familiar smells with their eyes closed. This can be done with } \\
\text { the help of an assistant who presents the odours to the patient. }\end{array}$ \\
\hline CN II & $\begin{array}{l}\text { - Physician should instruct patients to bring their eyes closer to the camera. } \\
\text { - Observe the pupils, noting symmetry and size. } \\
\text { - To test the pupillary light reflex, physician can ask patient to close their eyes for a few seconds then open them, noting } \\
\text { appropriate constriction. Similarly, the patient or assistant can use a flashlight. } \\
\text { - Assess visual fields by asking patient to cover each eye and describe what they see. Physician can print out the NIH stroke scale } \\
\text { - } \text { - Red desaturation can be tested by holding up a red object in front of patients. }\end{array}$ \\
\hline CN III, IV, VI & $\begin{array}{l}\text { - Physician should have the patient gaze in the } 9 \text { cardinal positions, with a brief pause at each position, looking for nystagmus and } \\
\text { ptosis. } \\
\text { - Assess for saccades by having the patient alternate their gaze between two extremes of their screens. }\end{array}$ \\
\hline $\mathrm{CN} \vee$ & $\begin{array}{l}\text { - Physician should observe the clenching and releasing of the jaw. } \\
\text { - Ask for areas of facial numbness or tingling. }\end{array}$ \\
\hline CN VII & $\begin{array}{l}\text { - Physician should have the patient smile, show teeth, raise their eyebrows, squeeze eyes shut and purse their lips, observing for } \\
\text { asymmetry. }\end{array}$ \\
\hline CN VIII & $\begin{array}{l}\text { - Physician should assess the patient's hearing ability by speaking to them in their normal voice. } \\
\text { - Assistant may test finger rub and note any asymmetry. }\end{array}$ \\
\hline $\mathrm{CN} \mathrm{IX}$ and $\mathrm{X}$ & $\begin{array}{l}\text { - Physician should watch for vocal abnormalities such as hoarseness. } \\
\text { - Physician can ask patients to open their mouth, say "ah" and assess for appropriate palatal elevation. }\end{array}$ \\
\hline $\mathrm{CN} \times \mathrm{I}$ & - Physician should ask patients to shrug their shoulders and rotate their neck. \\
\hline CN XII & eir tongue, noting any unilateral deviation, atrophy or fasciculations. \\
\hline
\end{tabular}

Notes: ${ }^{a}$ Data from Verduzco-Gutierrez et al. ${ }^{18}$ Al Hussona et al ${ }^{19}$ and from an American Headache Society video by Dr. J. Robblee. ${ }^{20}$

Abbreviations: $\mathrm{CN}$, cranial nerve; $\mathrm{NIH}$, National Institutes of Health.

used by headache patients. ${ }^{22}$ They allow for a clearer diagnosis, better assessment of burden, accurate medication use and therapy response. They can also improve medication adherence and help identify triggers and timing of attacks. ${ }^{22}$ When the patient self-reports lifestyles accurately, they can then identify behaviors that act as precursors in prodromal headache exacerbation stages (eg medication overuse and stress).

Many applications have been validated for the management of migraine attacks but unfortunately, some are no longer available for download. Despite this, examining user satisfaction and adaptability to such applications serves as a reflection of the overall usefulness of this type of clinical tool. Huguet et al developed an application called myWHI which allowed users to report occurrence (start time, initial intensity, starting location, potential triggers and prior symptoms), as well as describe the end of their headache (time, quality, peak, pain intensity level change in location and alleviating factors). ${ }^{17}$ Patients could also enter additional information like mood, sleep and impact of headache on daily activities. Sixty-five participants aged 14 to 28 tested the diary for 14 days and found it to be useful, easy to learn and efficient to use. Limitations found in this study include that the app was used by patients for only 14 days which is considered insufficient as opposed to an optimal trial period of 28 days. Additionally, the target population's age was quite narrow and therefore results cannot be generalized to older adults with similar symptoms.

Comparably, another application that was developed without a commercial use in mind intended to identify triggers in 62 migraine patients logging entries for 3 months. ${ }^{23}$ The most common trigger factors present on headache days were stress, sleep deprivation, fatigue, 
hormonal and weather changes. Headaches linked to identifiable triggers were more severe and more resembling of a migraine. Triggers that were less frequent included sunlight, odors, cheese, chocolate and fasting. Researchers found that apps can be successful in identifying triggers for migraine attacks. A rapid clinical implication of this research is that patients can avoid triggers that are specific to them instead of avoiding triggers that are generally listed as potential, thereby improving the patients' quality of life. While this app is not commercially available, this paper sheds light on the importance of developing a personalized trigger detection app or at least improving the logging of triggers in existing migraine diary apps.

The iHeadache application is currently available for download for the purpose of reporting symptoms related to headaches. ${ }^{24}$ Out of 106 participants giving their opinion on the app, $71 \%$ said it changed their headache care and $90 \%$ found it more practical than paper-based diaries. An evaluation of headache applications available for download on AppStore and Google Play was conducted. $^{25}$ Their customizability, clinical accuracy, design efficacy and user engagement were compared and the top three applications were, in order, Migraine Buddy, Migraine Coach and Migraine Monitor. Migraine Buddy records and identifies migraine triggers, symptoms, medication, frequency, duration, pain intensity and location. It can also provide a summary report to send to physicians and to help the patient better understand their headache. Lastly, it features a sleep diary to see if a correlation can be established between the patient's headache and sleeping pattern. Despite these numerous potentially useful functionalities, the authors found that they may represent too much information that might not always be relevant to patients. ${ }^{25}$ Migraine Coach allows patients to log in their symptoms, triggers and medication. This application is even light-sensitive to make it easier for patients to use during a migraine episode. ${ }^{25}$ The application also features a chat which uses artificial intelligence to help answer some of the most common questions about headaches. However, it does not provide reports like Migraine Buddy, which makes it more difficult for patients to understand their headache. ${ }^{25}$ Lastly, Migraine Monitor can help patients track their headache symptoms, triggers and medication. It allows physicians to directly visualize their patient's inputs. However, it was found to be less userfriendly than the two other applications, mostly because $\log$ entries could easily be deleted and had to be re-entered if the user was to change tab while recording an attack. ${ }^{25}$
Therefore, all three of these applications are appropriate for use in the telemedicine context, with Migraine Buddy capturing the most detailed clinical picture.

Development and production of wearables (wireless body sensors) to predict headaches is currently being explored. ${ }^{21}$ It will be interesting to see how this field evolves given the incentive to invest in telemedical technologies during the current pandemic context.

Despite the apparent advantages of incorporating mHealth tools into clinical practice, their limitations must be borne in mind as well. There are currently over 120000 mobile medical apps available on app stores, but over $80 \%$ of them have been created without early or long-standing involvement of patients, medical professionals or representatives of the scientific community. ${ }^{22}$ When it comes to headache diary apps specifically, it has been reported that only $18 \%$ of them have been created with scientific or clinical expertise. ${ }^{21}$ Although migraine is the $3^{\text {rd }}$ most common condition for which health applications are used, it is also the least researched. ${ }^{21}$ This could imply that the development of mHealth tools for migraine has more of a commercial and economic incentive than a scientific one. ${ }^{21}$ It would therefore be important to make sure that apps chosen for use in the clinical context are based on medical evidence currently available and are developed in conjunction with experts in the field. Moreover, mobile diary apps are not necessarily easy or fast to adapt into a daily routine. ${ }^{8}$ Physicians could thus plan for an adaptation period of at least 28 days at the beginning of their usage. ${ }^{17}$ We suggest that physicians also ensure that patients have been well-informed on how to use the applications. They should review the data with their patients and provide guidance on how to interpret the generated reports. Lastly, for optimal results, physicians should not neglect the therapeutic relationship with their patients when providing virtual care. They can do so by ensuring adequate follow-up and being vigilant not to solely rely on information from the migraine apps to assess their patients' headaches.

\section{Telemedicine vs Face-to-Face Consultations}

There is evidence to conclude that telemedicine has noninferior patient outcomes when compared to traditional face-to-face appointments for headaches. ${ }^{14-16}$ To demonstrate non-inferiority, authors have to define a clinically relevant "non-inferiority margin" for the size of any 
decrements in treatment effect that are considered not to be relevant. All three studies used non-inferiority margins of $15 \%$ meaning that outcome measures used for telemedicine appointments differed no more than $15 \%$ compared to those for in-person appointments. For instance, Bekkelund et al conducted an RCT with patients suffering from medication overuse headaches (MOH). ${ }^{16}$ They assigned half of their 102 participants to a videoconference appointment, and the other half to traditional face-to-face consultations. In both groups, headache burden was measured using HIT6 , frequency of headache days and VAS. There was no significant difference in HIT-6 and VAS between the groups measured prospectively at baseline, 3 months and 1 year. The effect of video consultations was deemed to be non-inferior to traditional consults with respect to the treatment responses and long-term treatment outcomes of patients with $\mathrm{MOH}$. As mentioned in the Assessment Scores section, Müller et al published two studies on a randomized trial of patients referred to a neurologist for chronic headache. ${ }^{14,15}$ They assigned 200 participants to videoconference and 202 participants to a traditional consultation. Three and twelve months after the initial consultation, VAS and HIT-6 scores were not statistically different ${ }^{14,15}$ and patient satisfaction was similar in both groups. ${ }^{14}$ Patients' headache status and variables of treatment efficiency also did not differ when compared to baseline. The authors were then able to provide evidence that telemedicine is non-inferior to traditional consultations in patient satisfaction, change in headache status, treatment efficiency and compliance.

Similarly, another study, while not proving noninferiority explicitly due to inadequate power, deemed telemedicine to be as effective as traditional consultations with respect to certain outcome measures. In this study, Friedman et al delivered treatment to 18 migraine patients via videoconference and to 12 patients via face-to-face appointments. ${ }^{8}$ Clinical outcomes in telemedicine, including MIDAS score, number of headache days per month and average severity of headaches, were not statistically different from those in the traditional group.

Offering healthcare over telemedicine has not only proven to be non-inferior in some studies, but it also comes with other advantages when compared to traditional clinical appointments. These advantages are summarized in Table 2 along with some limitations. First, convenience was rated higher in migraine telemedicine consultations. ${ }^{8,26}$ This was based on patient's appreciation of avoided travel time, less disruption of their daily routine and feeling more comfortable in the environment of their choice. Some studies found that the mean total time spent in a videoconference appointment was much shorter than the time spent around an in-person appointment. ${ }^{8,27}$ As an example, one study found that telemedicine and in-person appointments took on average 37 and 194 minutes, respectively. ${ }^{8}$ Of note, it must be disclosed that the calculation of the length of the in-person consult included the travel time and additional steps required prior to seeing the physician in office, such as check-in, taking vital signs, update of medication list and rooming. This also implies that physicians can see more patients and therefore make better use of their time. When excluding travel time and waiting times, telemedicine appointments were found to be shorter on average than traditional visits, being 38.8 and 43.7 minutes, respectively. ${ }^{27}$ Telemedicine also increases access to healthcare, especially to patients living in rural areas and reduces the costs associated with healthcare appointments (lost work opportunity, travel and parking costs). ${ }^{26,27}$

Patients' satisfaction with the clinical care provided via telemedicine is key to it becoming an accepted routine

Table 2 Advantages and Limitations of Telemedicine for Non-Acute Headaches

\begin{tabular}{|c|c|}
\hline Advantages & Limitations \\
\hline $\begin{array}{l}\text { - Evidence of non-inferiority when compared to in-person consultations }{ }^{14-16} \\
\text { - Convenient (eg, avoided travel time, less disruption of daily routine, more } \\
\text { comfortable setting) } \\
\text { - Increased access to specialized care in remote areas }{ }^{8,27} \\
\text { - Cost-effective } \\
\text { - Timesaving for healthcare providers } \\
\text { - High levels of patient satisfaction } \\
\text { - H, } 12,14,26,27\end{array}$ & $\begin{array}{l}\text { - Could increase disparity in healthcare delivery to rural and } \\
\text { minority groups }{ }^{12,29,30} \\
\text { - Limited power of physician observation } \\
\text { - Lack of face-to-face interaction between patient and physician } \\
\text { - Medicolegal liability is the same as with in-person visits }{ }^{81} \\
\text { - Certain level of technological knowledge necessary }\end{array}$ \\
\hline
\end{tabular}

Abbreviations: IHS, International Headache Society; PICO, population, intervention, comparison, outcome; RCT, randomized control trial; VAS, visual analog scale; HIT-6, headache impact test 6; MIDAS, migraine disability assessment; IMMPACT, initiative on methods, measurement, and pain assessment in clinical trials; RAPD, relative afferent pupillary defect; $\mathrm{MOH}$, medication overuse headache. 
clinical tool. Several studies have demonstrated a high level of satisfaction with telemedicine visits from headache patients $8,12,14,26,27$ and others have shown patients' satisfaction rates to be equal in both telemedicine and traditional consultations. ${ }^{8,14}$ Given this, there are several recommendations that, when implemented, can be conducive to increased satisfaction. ${ }^{28}$ Before the visit takes place, physicians should set realistic expectations of the interview. This can be done by informing the patient what the interview will consist of and by asking the patient if there is anything specific they would like to get out of the interview. The importance of setting these expectations is to minimize deviations from expected care regardless of the quality of the visit or objective medical outcomes. Lastly, physicians should not only employ easy to use and reliable technology, but also select patients who are adaptable and are likely to welcome the convenience of this technology.

\section{Limits of Telemedicine}

We must also be cognizant of the limitations of telemedicine, both from a patient and physician perspective. First, since telemedicine mostly depends on technology, it could further increase disparity in the delivery of quality healthcare, primarily impacting rural communities, racial and ethnic minorities as well as low-income populations. For instance, some rural regions may not have access to a reliable internet connection due to lack of infrastructure or to mobile technologies such as smartphones. ${ }^{29}$ Additionally, racial and ethnic minority groups tend to have more telephone than videoconference consultations, suggesting a lack of access. ${ }^{12}$ Groups with lower household income were shown to be less likely to attend telemedicine appointments. ${ }^{30}$ To add to this, the COVID-19 pandemic is preventing already underserved minority groups from accessing neurological care. ${ }^{30}$ Physicians should also prioritize applications that are culturally and linguistically appropriate for the patient population they serve. Although Migraine Buddy is available in nine different languages, iHeadache and Migraine Coach are only available in English. Another recurrent concern is that telemedicine limits physicians' power of observation. However, in migraine care, a thorough history is usually sufficient to make the diagnosis and follow-up care does not generally necessitate in-person physical exam or intervention. ${ }^{7,8}$ Nonetheless, physicians new to telemedicine may not be able to extract the same amount of information due to difficulty of interpreting body language and reduced back-and-forth discussion. This lack of direct interaction with the patient may figuratively distance the physician even more and clinicians may find it difficult to stay focused on what is being said in the interview.

As with any other medical encounter, the physician's medicolegal liability is the same as with in-person visits, which can be perceived as a disadvantage for physicians unable to fully examine their patients in person. ${ }^{31}$ In addition, some patients may prefer to be examined faceto-face rather than over videoconference. ${ }^{8}$ Other negatives as reported by patients were the level of technological knowledge necessary to engage in a videoconference appointment, the inability to get urgent treatment and having to obtain vital signs elsewhere. ${ }^{8}$

\section{Pandemic Considerations}

The proven validity of performing headache consultations over telemedicine represents a considerable asset in the context of the pandemic given the heightened risk of inperson encounters. Although physicians have a convenient tool to adapt their practice, they must take into account new challenges the pandemic has introduced. Firstly, clinicians should be aware that COVID19 infection may result in new onset headaches or can worsen pre-existing primary headache disorders. ${ }^{7}$ In the context of headache management specifically, it is important to mention that lockdown can increase exposure to triggers commonly affecting migraine patients. ${ }^{7}$ In return, this can lead to higher frequency of headache attacks, increased medication usage and, therefore, increased $\mathrm{MOH}$. Consequently, physicians might be seeing more patients with headaches than they did prior to the COVID-19 pandemic and must make the necessary adaptations to take on a potentially larger number of appointments. It is recommended that patients try to stick to a routine as much as possible and be referred to mental health resources when struggling with lockdown burden. ${ }^{7}$ Additionally, physicians can suggest modifying at-home work environment such as lighting, screen brightness, seating, odors or heat exposure in an effort to minimize headache triggers.

\section{Conclusion}

Studies have demonstrated that telemedicine for headaches is feasible, practical and has been accepted by both physicians and patients. In this review, we have outlined the components of a remote assessment for headaches which includes patient selection, assessment scores and neurological exam. Upon setting a triaging 
system to identify those cases appropriate for remote consultation and those for in-person visits, a wellstructured virtual consultation has been shown to be non-inferior to its face-to-face counterpart and presents advantages to both patients and physicians. These include high satisfaction, convenience and cost-saving, especially in rural populations. Lastly, mHealth apps for headache documentation such as Migraine Buddy, Migraine Coach and Migraine Monitor have been shown to be useful in improving communication between patients and physicians. A balance between the amount of data collected by the app for clinical purposes and the patient's perception of satisfaction must be found. Likewise, physicians should allow for an adjustment period when introducing a migraine app to their patients and ensure that they have been wellinformed on how to use the app. Overall, telemedicine is a well-suited technology for managing non-acute headache as it is comparable to in-office treatment, costeffective and we believe that its advantages outweigh its limitations especially for populations who lack ready access to a specialist.

\section{Acknowledgments}

The research activities of the Edwards Family Interdisciplinary Complex Pain Centre of the Montreal Children's Hospital are supported by unrestricted grants of the Montreal Children's Hospital Foundation and of the Louise and Alan Edwards Foundation.

\section{Disclosure}

Dr. Ingelmo received unrestricted support from the Louise and Alan Edwards Foundation and from the Montreal Children's Hospital Foundation. The authors report no other conflicts of interest in this work.

\section{References}

1. Headache Classification Committee of the International Headache Society (IHS) The International Classification of Headache Disorders, 3rd edition. Cephalalgia. 2018;38(1):1-211. doi:10.1177/ 0333102417738202

2. Vos T, Abajobir AA, Abate KH, et al. Global, regional, and national incidence, prevalence, and years lived with disability for 328 diseases and injuries for 195 countries, 1990-2016: a systematic analysis for the Global Burden of Disease Study 2016. The Lancet. 2017;390 (10100):1211-1259.

3. Howard IM, Kaufman MS. Telehealth applications for outpatients with neuromuscular or musculoskeletal disorders. Muscle Nerve. 2018;58 (4):475-485.
4. Bhaskar S, Nurtazina A, Mittoo S, Banach M, Weissert R. Editorial: telemedicine during and beyond COVID-19. editorial. Front Public Health. 2021;9(233). doi:10.3389/fpubh.2021.662617

5. Bhaskar S, Bradley S, Israeli-Korn S, et al. Chronic neurology in COVID-19 era: clinical considerations and recommendations from the REPROGRAM Consortium. Front Neurol. 2020;11:664. doi:10.3389/fneur.2020.00664

6. Garg S, Gangadharan N, Bhatnagar N, Singh MM, Raina SK, Galwankar S. Telemedicine: embracing virtual care during COVID-19 pandemic. J Family Med Prim Care. 2020;9 (9):4516-4520. doi:10.4103/jfmpc.jfmpc_918_20

7. Chowdhury D, Datta D. Managing migraine in the times of COVID-19 pandemic. Ann Indian Acad Neurol. 2020;23(Suppl 1): S33.

8. Friedman DI, Rajan B, Seidmann A. A randomized trial of telemedicine for migraine management. Cephalalgia. 2019;39 (12):1577-1585.

9. Sinha S, Kern LM, Gingras LF, et al. Implementation of video visits during COVID-19: lessons learned from a primary care practice in New York city. Front Public Health. 2020;8:514. doi:10.3389/ fpubh.2020.00514

10. Bhaskar S, Bradley S, Chattu VK, et al. Telemedicine across the globe-position paper from the COVID-19 pandemic health system resilience PROGRAM (REPROGRAM) International Consortium (Part 1). Front Public Health. 2020;8:556720. doi:10.3389/ fpubh.2020.556720

11. Rush KL, Howlett L, Munro A, Burton L. Videoconference compared to telephone in healthcare delivery: a systematic review. Int $J \quad$ Med Inform. 2018;118:44-53. doi:10.1016/j. ijmedinf.2018.07.007

12. Rametta SC, Fridinger SE, Gonzalez AK, et al. Analyzing 2,589 child neurology telehealth encounters necessitated by the COVID-19 pandemic. Neurology. 2020.

13. Dodick DW, Tepper SJ, Lipton RB, et al. Improving medical communication in migraine management: a Modified Delphi study to develop a digital migraine tracker. Headache. 2018;58(9):1358-1372.

14. Müller KI, Alstadhaug KB, Bekkelund SI. Telemedicine in the management of non-acute headaches: a prospective, open-labelled non-inferiority, randomised clinical trial. Cephalalgia. 2017;37 (9):855-863

15. Müller KI, Alstadhaug KB, Bekkelund SI. A randomized trial of telemedicine efficacy and safety for nonacute headaches. Neurology. 2017;89(2):153-162.

16. Bekkelund SI, Müller KI. Video consultations in medication overuse headache. A randomized controlled trial. Brain Behav. 2019;9(7): e01344.

17. Huguet A, McGrath PJ, Wheaton M, et al. Testing the feasibility and psychometric properties of a mobile diary (myWHI) in adolescents and young adults with headaches. JMIR mHealth uHealth. 2015;3(2): e39.

18. Verduzco-Gutierrez M, Bean AC, Tenforde AS, Tapia RN, Silver JK. How to Conduct an outpatient telemedicine rehabilitation or prehabilitation visit. $P M \&$ R. 2020.

19. Al Hussona M, Maher M, Chan D, et al. The Virtual neurologic exam: instructional videos and guidance for the COVID-19 era. Can J Neurol Sci. 2020;47(5):598-603. doi:10.1017/cjn.2020.96

20. Robblee J. Conducting a Telemedicine Neurologic Examination. American Headache Society; 2020. Available from: https://american headachesociety.org/news/telemedicine-neurologic-examination/. Accessed February 23, 2021.

21. Stubberud A, Linde M. Digital technology and mobile health in behavioral migraine therapy: a narrative review. Curr Pain Headache Rep. 2018;22(10):66.

22. van de Graaf DL, Schoonman GG, Habibović M, Pauws SC. Towards eHealth to support the health journey of headache patients: a scoping review. J Neurol. 2020;1-20. 
23. Park J-W, Chu MK, Kim J-M, Park S-G, Cho S-J. Analysis of trigger factors in episodic migraineurs using a smartphone headache diary applications. PLoS One. 2016;11(2):e0149577.

24. Mosadeghi-Nik M, Askari MS, Fatehi F. Mobile health (mHealth) for headache disorders: a review of the evidence base. $J$ Telemed Telecare. 2016;22(8):472-477.

25. Nguyen V, Pergami P Applications Geared Towards Chronic Migraines: Content Analysis.

26. Qubty W, Patniyot I, Gelfand A. Telemedicine in a pediatric headache clinic: a prospective survey. Neurology. 2018;90(19):e1702e1705.

27. Müller KI, Alstadhaug KB, Bekkelund SI. Acceptability, feasibility, and cost of telemedicine for nonacute headaches: a randomized study comparing video and traditional consultations. $J$ Med Internet Res. 2016;18(5):e140. doi:10.2196/jmir.5221
28. Nguyen M, Waller M, Pandya A, Portnoy JA. Review of patient and provider satisfaction with telemedicine. Curr Allergy Asthma Rep. 2020;20(11):72. doi:10.1007/s11882-020-00969-7

29. Davis LE, Harnar J, LaChey-Barbee LA, Pirio Richardson S, Fraser A, King MK. Using teleneurology to deliver chronic neurologic care to rural veterans: analysis of the first 1100 patient visits. Telemed e-Health. 2019;25(4):274-278.

30. Nolen L, Mejia NI. Inequities in neurology amplified by the COVID-19 pandemic. Nat Rev Neurol. 2021;17(2):67-68. doi:10.1038/s41582-020-00452-x

31. Lo MD, Gospe SM Jr. Telemedicine and child neurology. J Child Neurol. 2019;34(1):22-26.

\section{Publish your work in this journal}

The Journal of Pain Research is an international, peer reviewed, open access, online journal that welcomes laboratory and clinical findings in the fields of pain research and the prevention and management of pain. Original research, reviews, symposium reports, hypothesis formation and commentaries are all considered for publication. The manuscript management system is completely online and includes a very quick and fair peer-review system, which is all easy to use. Visit http:// www.dovepress.com/testimonials.php to read real quotes from published authors. 